\title{
Identification of Dark Matter with directional detection
}

\section{J. Billard*, F. Mayet, D. Santos}

Laboratoire de Physique Subatomique et de Cosmologie, Université Joseph Fourier Grenoble 1, CNRS/IN2P3, Institut Polytechnique de Grenoble, Grenoble, France

E-mail: billardelpsc.in2p3.fr

Directional detection is a promising search strategy to discover galactic Dark Matter. Taking advantage on the rotation of the Solar system around the Galactic center through the Dark Matter halo, it allows to show a direction dependence of WIMP events. Data of directional detectors are composed of energy and a 3D track for each recoiling nuclei. Here, we present a Bayesian analysis method dedicated to data from upcoming directional detectors. However, we focus only on the angular part of the event distribution, arguing that the energy part of the background distribution is unknown. Two different cases are considered: a positive or a null detection of Dark Matter. In the first scenario, we will present a map-based likelihood method allowing to recover the main incoming direction of the signal and its significance, thus proving its Galactic origin. In the second scenario, a new statistical method is proposed. It is based on an extended likelihood in order to set robust and competitive exclusion limits. This method has been compared to two other methods and has been shown to be optimal in any detector configurations. Eventually, prospects for the MIMAC project are presented in the case of a $10 \mathrm{~kg} \mathrm{CF}_{4}$ detector with an exposition time of 3 years.

Identification of Dark Matter 2010-IDM2010

July 26-30, 2010

Montpellier France

\footnotetext{
${ }^{*}$ Speaker.
} 


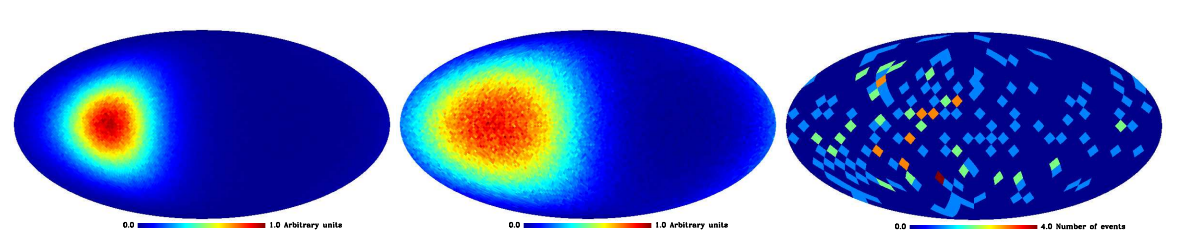

Figure 1: From left to right: WIMP flux in the case of an isothermal spherical halo, WIMP-induced recoil distribution and a typical simulated measurement : 100 WIMP-induced recoils and 100 background events with a low angular resolution. Recoil maps are produced for a Fluorine target, a $100 \mathrm{GeV} \cdot \mathrm{c}^{-2}$ WIMP and considering recoil energies in the range $5 \mathrm{keV} \leq E_{R} \leq 50 \mathrm{keV}$. Figures are taken from [7].

\section{Directional detection framework}

Taking advantage of the astrophysical framework, directional detection of Dark Matter is an interesting strategy in order to distinguish WIMP events from background ones. Indeed, like most spiral galaxies, the Milky Way is supposed to be immersed in a halo of WIMPs which outweighs the luminous component by at least one order of magnitude. As the Solar System rotates around the galactic center through this Dark Matter halo, WIMPs should mainly come from the direction to which points the Sun velocity vector and which happens to be roughly in the direction of the Cygnus constellation. Then, a directional WIMP flux is expected to enter any terrestrial detectors (see fig.1 left) infering a directional WIMP induced recoil distribution which should be pointing toward the Cygnus Constellation, i.e. in the $\left(\ell_{\odot}=90^{\circ}, b_{\odot}=0^{\circ}\right)$ direction (see fig. 1 middle). The latter corresponds to the expected WIMP signal probed by directional detectors and as it is shown on the fig. 1 (middle), a strong anisotropy is expected [1] while the background should be isotropic. Hence, we argue that a clear and unambigous identification of a Dark matter detection could be done by showing the correlation of the measured signal with the direction of the solar motion.

Several project of directional detectors are being developed [2,3] and some of them are already taking data $[4,5,6]$. We present a complete analysis framework dedicated to directional data. The first step when analysing directional data should be to looking for a signal pointing toward the Cygnus Constellation with a sufficiently high significance. If no evidence in favor of a Galactic origin of the signal is deduced from the previous analysis, then an exclusion limit should be derived. Here we will discussed the two different cases considering a $10 \mathrm{~kg}$ of $\mathrm{CF}_{4}$ detector with a recoil energy range of $5 \mathrm{keV} \leq E_{R} \leq 50 \mathrm{keV}$.

\section{Case of positive detection}

An observed recoil map such as the one on the right of fig. 1 could be obtained by a $10 \mathrm{~kg} \mathrm{CF}_{4}$ detector with a WIMP-nucleon cross-section of $\sigma_{n}=1.5 \times 10^{-3} \mathrm{pb}$ and with a background rate of $\sim 0.07 \mathrm{~kg}^{-1}$. day ${ }^{-1}$ in $\sim 5$ months exposition time. At first sight, it seems difficult to conclude from this simulated recoil map that it does contain a fraction of WIMP events pointing towards the direction of the Solar motion. A likelihood analysis is developed in order to retrieve from a recoil map : the main direction of the incoming events in Galactic coordinates $(\ell, b)$ and the number of WIMP events contained in the map. The likelihood value is estimated using a binned map of the 
overall sky with Poissonian statistics, as follows :

$$
\mathscr{L}\left(m_{\chi}, \lambda, \ell, b\right)=\prod_{i=1}^{N_{\text {pixels }}} P\left(\left[(1-\lambda) B_{i}+\lambda S_{i}\left(m_{\chi} ; \ell, b\right)\right] \mid M_{i}\right)
$$

where $B$ is the background spatial distribution taken as isotropic, $S$ is the WIMP-induced recoil distribution and $M$ is the measurement. This is a four parameter likelihood analysis with $m_{\chi}, \lambda=S /(B+S)$ the WIMP fraction (related to the background rejection power of the detector) and the coordinates $(\ell, b)$ referring to the maximum of the WIMP event angular distribution. Hence, $S\left(m_{\chi} ; \ell, b\right)$ corresponds to a rotation of the $S\left(m_{\chi}\right)$ distribution by the angles $\left(\ell^{\prime}=\ell-\ell_{\odot}\right.$, $\left.b^{\prime}=b-b_{\odot}\right)$. A scan of the four parameters with flat priors, allows to evaluate the likelihood between the measurement (fig. 1 right) and the theoretical distribution made of a superposition of an isotropic background and a pure WIMP signal (fig. 1 middle). By scanning on $\ell$ and $b$ values, we ensure that there is no prior on the direction of the center of the WIMP-induced recoil distribution. As the observed map is considered as a superposition of the background and the WIMP signal distributions, no assumption on the origin of each event is needed. Moreover, the likelihood method allows to recover $\lambda$, the WIMP fraction contained in the data. The advantage is twofold :

- First, background-induced bias is avoided. This would not be the case with a method trying to evaluate a likelihood on a map containing a fairly large number of background events considering only a pure WIMP reference distribution.

- Second, the value of $\lambda$ allows to access the number of genuine WIMP events and consequently to constrain the WIMP-nucleon cross-section as presented in [7] and to estimate the significance of the Dark Matter detection.

The four parameter likelihood analysis has been computed on the simulated map (fig. 1 right) and the conclusion of this working example is the following; we found that it does contain a signal pointing towards the Cygnus constellation within $10^{\circ}$, with $N_{\text {wimp }}=106 \pm 17$ (68\%CL), corresponding to a high significance detection of Galactic Dark Matter.

In order to explore the robustness of the method, and to ensure that it gives satisfactory results on a large range of exposure and background contamination, a systematic study has been done with $10^{4}$ experiments for various number of WIMP events $\left(N_{\text {wimp }}\right)$ and several values of WIMP fraction in the observed map $(\lambda)$, ranging from 0.1 to 1 . For a given cross-section, these two parameters are related respectively with the exposure and the rejection power of the offline analysis preceding the likelihood method.

Figure 2 presents on the left panel the directional signature, taken as the value of $\sigma_{\gamma}=\sqrt{\sigma_{\ell} \sigma_{b}}$, the radius of the $68 \% \mathrm{CL}$ contour of the marginalised $\mathscr{L}(\ell, b)$ distribution, as a function of $\lambda$. It is related to the ability to recover the main signal direction and to sign its Galactic origin. It can first be noticed that the directional signature is of the order of $10^{\circ}$ to $20^{\circ}$ on a wide range of WIMP fractions. Even for low number of WIMPs and for a low WIMP fraction (meaning a poor rejection power), the directional signature remains clear. From this, we conclude that a directional evidence in favor of Galactic Dark Matter may be obtained with upcoming experiments even at low exposure (i.e. a low number of observed WIMPs) and with a non-negligible background contamination. 

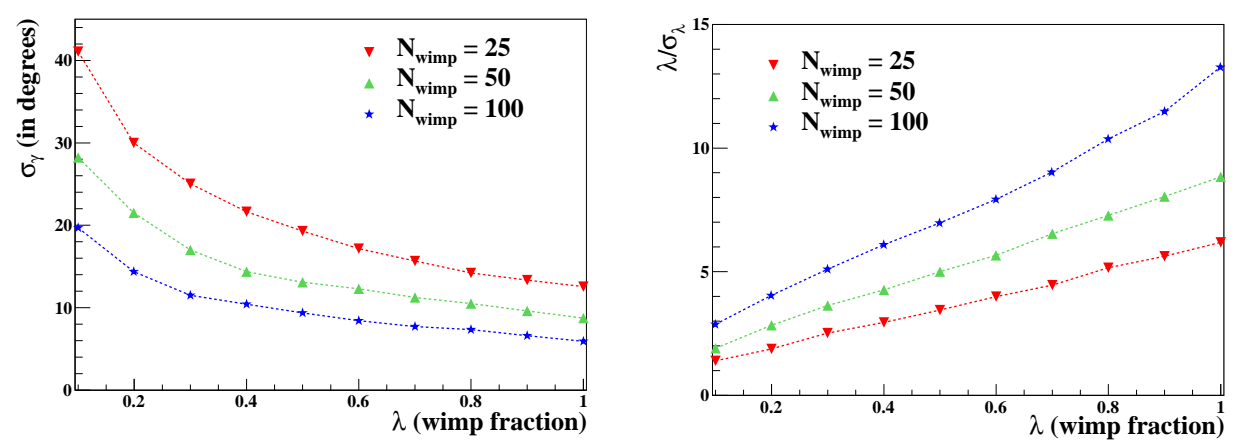

Figure 2: Left panel presents the directional signature $\sigma_{\gamma}$ (in degrees) as a function of the WIMP fraction. Right panel presents the significance $\left(\lambda / \sigma_{\lambda}\right)$ as a function of the WIMP fraction $\lambda=S /(S+B)$. Results are produced for a ${ }^{19} \mathrm{~F}$ target, a $100 \mathrm{GeV} \cdot \mathrm{c}^{-2}$ WIMP and considering recoil energies in the range $5 \mathrm{keV} \leq E_{R} \leq$ $50 \mathrm{keV}$. Figures are taken from [7].

However, a convincing proof of the detection of WIMPs would require a directional signature with sufficient significance. We defined the significance of this identification strategy as $\lambda / \sigma_{\lambda}$, presented on figure 2 (right panel) as a function of $\lambda=S /(S+B)$. As expected, the significance is increasing both with the number of WIMP events and with the WIMP fraction, but we can notice that an evidence $(3 \sigma)$ or a discovery $(5 \sigma)$ of a Dark Matter signal would require either a larger number of WIMPs or a lower background contamination.

Using this map-based likelihood method, a directional detector may provide, as a first step, a Galactic signature even with a low number of WIMPs. For instance, a signal pointing towards Cygnus within $20^{\circ}$ can be obtained with as low as 25 WIMPs with a $50 \%$ background contamination. For an axial cross-section on nucleon of $\sigma_{\mathrm{n}}=1.5 \times 10^{-3} \mathrm{pb}$, this corresponds to an exposure of 400 $\mathrm{kg}$.day in $\mathrm{CF}_{4}$. In a second step, with an exposure four times larger, the directional signature would be only slightly better $\left(10^{\circ}\right)$ but the significance would be much higher $(\sim 7 \sigma)$ and the detection much more convincing.

\section{Case of a null detection}

This map-based likelihood analysis tool gives satisfactory results on a large range of exposure and background contamination levels. But, for very low number of WIMP events and for very large background fractions $(\lambda \rightarrow 0)$, when this method failed at recognizing a WIMP detection, obviously an exclusion limit should be derived. This will be the case for the very first results of directional detectors with low exposures or if the WIMP-nucleon cross-section lies below $\sim 10^{-5} \mathrm{pb}$. Here, we present a Bayesian estimation of exclusion limits dedicated to directional data where only the angular part of the event distribution is considered. The fact that both signal and background angular spectra are well known allows to derive upper limits using the Bayes' theorem. Considering an extended likelihood function with flat priors for both the expected number of WIMP events $\left(\mu_{s}\right)$ and background events $\left(\mu_{b}\right)$, and taking the evidence as a normalization factor, it is reduced to

$$
P\left(\mu_{s}, \mu_{b} \mid \vec{D}\right) \propto \frac{\left(\mu_{s}+\mu_{b}\right)^{N}}{N !} e^{-\left(\mu_{s}+\mu_{b}\right)} \times \prod_{i=1}^{N_{\text {pixels }}} P\left(\frac{\mu_{s}}{\mu_{s}+\mu_{b}} S_{i}+\frac{\mu_{b}}{\mu_{s}+\mu_{b}} B_{i} \mid M_{i}\right)
$$


where $\vec{D}$ refers to the characteristics of the data, as the total number of recorded events $N$, their direction and their energy. It can be noticed that we use exactly the same likelihood definition than for the previous discovery method. Indeed, the $\lambda$ parameter has been splitted into $\mu_{s}$ and $\mu_{b}$ and the WIMP mass is fixed. Hence, the probability density function of the parameter of interest $\mu_{s}$ can be derived by marginalizing $P\left(\mu_{s}, \mu_{b} \mid \vec{D}\right)$ over the parameter $\mu_{b}$ and the excluded number of WIMP events $\mu_{\text {exc }}$, corresponding to an excluded cross-section, at $90 \%$ CL is obtained by solving:

$$
\int_{0}^{\mu_{\mathrm{exc}}} P\left(\mu_{s} \mid \vec{D}\right) d \mu_{s}=0.9
$$

We have also used two other statistical methods: the Poisson method and the maximum gap method [9] applied to directional data. For each detector configurations and inputs, we have used 10000 toy Monte Carlo experiments in order to evaluate the frequency distributions of the excluded crosssection. Then, from each distribution, we can derive the median value of the excluded cross-section $\sigma_{\text {med }}$ for the three statistical methods. More details may be found in [8].

We are interested on the effect of detector configurations on the excluded cross-section. Indeed, even though several progresses have been done, sense recognition and angular resolution remain challenging experimental issues for directional detection of Dark Matter.

Without sense recognition, a recoil coming from $(\cos \gamma, \phi)$ cannot be distinguished from a recoil coming from $(-\cos \gamma, \phi+\pi)$. The effect of no sense recognition in the case of pure background data is shown on figure 3 where it is compared with the case where the detector has a $100 \%$ efficiency in the sense recognition. First of all, we can see on figure 3 that the Likelihood method overcomes the two others in both cases. Secondly, the absence of sense recognition only mildly alter the result: a factor of three at high background contamination. Taken at face value, this result suggests that sense recognition may not be so important for directional detection when setting exclusion limits. The difference between $100 \%$ sense recognition on the whole recoil energy range, which is obviously unrealistic, and no sense recognition is only minor. The worst case is indeed a partial sense recognition strongly depending on the recoil energy. In this case, we suggest not to consider this information to set robust exclusion limits. However, sense recognition remains a key issue worth investigating, for WIMP discovery which is the ultimate goal of directional detection.

About the angular resolution, even if simulations show that straight line tracks may be 3D reconstructed with a rather small angular dispersion, realistic tracks in low pressure gaseous detectors would encounter a rather large angular dispersion. The lower is the recoil energy, the larger is the angular straggling. Hence, in the following we investigate the effect on exclusion limits of using a detector with a finite (realistic) angular resolution. Having a finite angular resolution means that a recoil initially coming from the direction $\hat{r}(\theta, \phi)$ is reconstructed as a recoil $\hat{r}^{\prime}\left(\theta^{\prime}, \phi^{\prime}\right)$ with a gaussian dispersion of width $\sigma_{\Theta}$. Figure 3 presents the cross section limit as a function of the angular resolution $\sigma_{\Theta}$ in the case of 10 expected background events and one expected WIMP event. It can be noticed that the Maximum Gap and the likelihood methods are only slightly dependent on the angular resolution of the detector. The deviation for the two methods is of the order of $30 \%$ from $\sigma_{\Theta}=0^{\circ}$ to $\sigma_{\Theta}=45^{\circ}$. Hence, as far as exclusion limits are concerned, the effect of angular resolution is relatively small. 

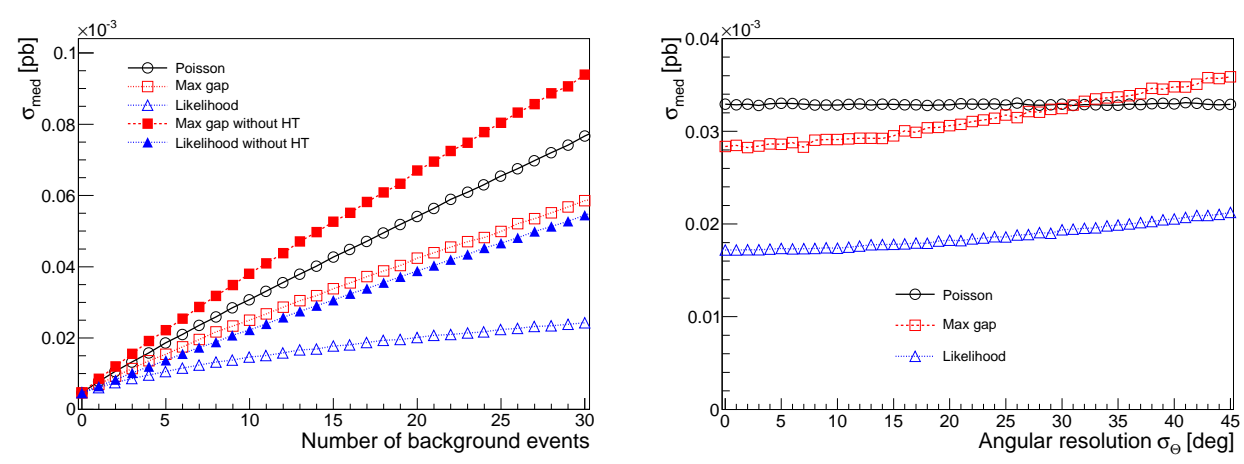

Figure 3: The median upper limit cross section obtained by the three different statistical methods as a function of the number of background events in the case of pure background (on the left) and as a function of the angular resolution (on the right). Left panel: The results without sense recognition are presented with filled markers while empty markers refer to a detector with sense recognition. Right panel: 10 expected background events and one expected WIMP event. Figures are taken from [8].

As a conclusion, this study outlines the need for detector commissioning, e.g. by using a neutron field. Indeed, in order to set coherent and robust upper limits, it is necessary to have an accurate knowledge of the detector characteristics, even if the effect on exclusion limits is small.

\section{Prospects for the $10 \mathrm{~kg} \mathrm{CF}_{4}$ MIMAC detector}

To end-up this study, we present projected discovery regions and exclusion limits for a forthcoming directional detector proposed by the MIMAC collaboration [3]. We consider a $10 \mathrm{~kg} \mathrm{CF}_{4}$ detector operated during $\sim 3$ years, allowing $3 \mathrm{D}$ track reconstruction, with a $10^{\circ}$ angular resolution, a recoil energy range $5-50 \mathrm{keV}$ and with a realistic background rate of $10 \mathrm{evts} / \mathrm{kg} / \mathrm{year}$. On figure 4 we have represented the two different possibilities for such a detector.

- The grey and light-grey shaded areas represent the contours where a detection of Dark Matter would have a significance greater than $3 \sigma$ and $5 \sigma$. These contours are deduced from the map-based likelihood method and its estimation of the significance. Then, as an illustration, if the WIMP-nucleon cross-section is about $10^{-4} \mathrm{pb}$ with a WIMP mass of $100 \mathrm{GeV} \cdot \mathrm{c}^{-2}$, the detector would have a Dark Matter detection with a significance greater than $3 \sigma$.

- If the WIMP-nucleon cross-section is lower than $10^{-5} \mathrm{pb}$, then an exclusion limit is deduced using the extended likelihood function (black dashed line). As a benchmark and to illustrate the effect of background on exclusion limits, we have also represented the detector sensitivity corresponding to the case where no events are recorded (black solid line).

Figure 4 presents exclusion limits from direct detection experiments, KIMS [10] and Picasso [11] as well as the theoretical region, obtained within the framework of the constrained minimal supersymmetric model taken from [12]. We can conclude that a directional detector like MIMAC will cover an important region of interest worth being investigated. 


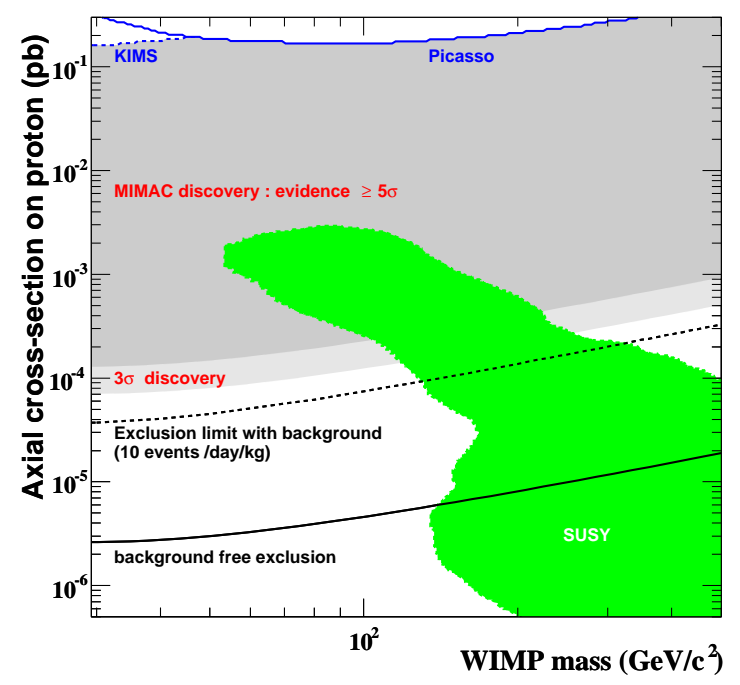

Figure 4: Spin dependent cross-section on proton (pb) as a function of the WIMP mass $\left(\mathrm{GeV} / \mathrm{c}^{2}\right)$. Exclusion limits from some direct detection experiments are presented, KIMS [10] and Picasso [11] as well as the theoretical region, obtained within the framework of the constrained minimal supersymmetric model taken from [12]. Contours corresponding to a significance greater than $3 \sigma$ and $5 \sigma$ are represented in grey and light grey. The exclusion limit corresponding to pure background data is represented as the black dashed line and the detector sensitivity as the black solid line.

This final study highlights the fact that directional detection should provide either an unambiguous signature in favor of a detection of Dark Matter if the cross section is reachable by such a detector or to set robust and competitive exclusion limits.

\section{References}

[1] D. N. Spergel, Phys. Rev. D 37 (1988) 1353

[2] S. Ahlen et al., International Journal of Modern Physics A25 (2010) 1-51

[3] C. Grignon et al., arXiv:1008.4712

[4] S. Ahlen et al., arXiv:1006.2928

[5] G. J. Alner et al., Nucl. Instr. Meth. A 555 (2005) 173.

[6] K. Miuchi et al., Phys. Lett. B 654 (2007) 58

[7] J. Billard, F. Mayet, J. F. Macias-Perez and D. Santos, Phys. Lett. B 691 (2010) 156-162

[8] J. Billard, F. Mayet and D. Santos, Phys. Rev. D 82 (2010) 055011

[9] S. Yellin, Phys. Rev. D 66 (2002) 032005

[10] H. S. Lee et al., Phys. Rev. Lett. 99 (2007) 091301

[11] S. Archambault et al., Phys. Lett. B 682 (2009) 185

[12] R. Trotta, F. Feroz, M. P. Hobson, L. Roszkowski and R. Ruiz de Austri, JHEP 0812 (2008) 024 